\title{
DNA methylation: the pivotal interaction between early-life nutrition and glucose metabolism in later life
}

\author{
Jia Zheng, Xinhua Xiao*, Qian Zhang and Miao Yu \\ Key Laboratory of Endocrinology, Department of Endocrinology, Ministry of Health, Peking Union Medical College Hospital, \\ Diabetes Research Center of Chinese Academy of Medical Sciences and Peking Union Medical College, No. 1 Shuaifuyuan, \\ Wangfujing Street, Dongcheng District, Beijing 100730, People's Republic of China
}

(Submitted 26 March 2014 - Final revision received 24 July 2014 - Accepted 7 August 2014 - First published online 20 October 2014)

\section{Abstract}

Traditionally, it has been widely acknowledged that genes together with adult lifestyle factors determine the risk of developing some metabolic diseases such as insulin resistance, obesity and diabetes mellitus in later life. However, there is now substantial evidence that prenatal and early-postnatal nutrition play a critical role in determining susceptibility to these diseases in later life. Maternal nutrition has historically been a key determinant for offspring health, and gestation is the critical time window that can affect the growth and development of offspring. The Developmental Origins of Health and Disease (DOHaD) hypothesis proposes that exposures during early life play a critical role in determining the risk of developing metabolic diseases in adulthood. Currently, there are substantial epidemiological studies and experimental animal models that have demonstrated that nutritional disturbances during the critical periods of early-life development can significantly have an impact on the predisposition to developing some metabolic diseases in later life. The hypothesis that epigenetic mechanisms may link imbalanced early-life nutrition with altered disease risk has been widely accepted in recent years. Epigenetics can be defined as the study of heritable changes in gene expression that do not involve alterations in the DNA sequence. Epigenetic processes play a significant role in regulating tissue-specific gene expression, and hence alterations in these processes may induce longterm changes in gene function and metabolism that persist throughout the life course. The present review focuses on how nutrition in early life can alter the epigenome, produce different phenotypes and alter disease susceptibilities, especially for impaired glucose metabolism.

\section{Key words: Epigenetics: DNA methylation: Early-life nutrition: Glucose metabolism}

Nowadays, the prevalence of diabetes mellitus is increasing extremely rapidly worldwide, which is now considered a pandemic non-communicable disease. According to the Diabetes Atlas published by the International Diabetes Federation ${ }^{(1)}$ on World Diabetes Day in 2013, 382 million people are suffering from diabetes and the number will rise to 592 million by 2035, which implies that one-tenth of the world's population will endure diabetes. In particular, more than 21 million infants inherited diabetes from their mothers during pregnancy in 2013. It is estimated that diabetes caused $5 \cdot 1$ million deaths in 2013 , i.e. a person died from the disease every $6 \mathrm{~s}$. In summary, diabetes is becoming a more and more severe problem for our society.

Unfortunately, the pathogenesis of diabetes has not been clearly understood yet. However, it is generally believed that genes together with adult lifestyle factors are the major risks of diabetes. There is substantial evidence that the prenatal and early-postnatal nutrition play a key role in determining susceptibility to diabetes in later life $\mathrm{e}^{(2)}$. Little is known about the molecular mechanisms underlying the interaction between maternal diet and ageing. However, the hypothesis that epigenetic mechanisms may link such imbalanced nutrition with altered disease risk has been widely accepted in recent years. Epigenetics is one of the major mechanisms explaining the theory of Developmental Origins of Health and Disease (DOHaD), which focuses on the association between perinatal nutrition and late-onset diseases, such as obesity, insulin resistance, impaired glucose tolerance and type 2 diabetes mellitus (T2DM) ${ }^{(3)}$. Therefore, epigenetics is likely to be an important molecular basis of malnutrition during early life and glucose metabolism disorders in later life (Fig. 1).

\section{An overview of epigenetics}

Traditionally, epigenetics has been used to explain the phenotypic events that cannot be explained by genetic mechanisms.

Abbreviations: DMR, differentially methylated region; $\mathrm{F}_{1}$, first-generation; $\mathrm{F}_{2}$, second-generation; GDM, gestational diabetes mellitus; GR, glucocorticoid receptor; IGF2, insulin-like growth factor 2; LP, low-protein; MZ, monozygotic; T2DM, type 2 diabetes mellitus. 


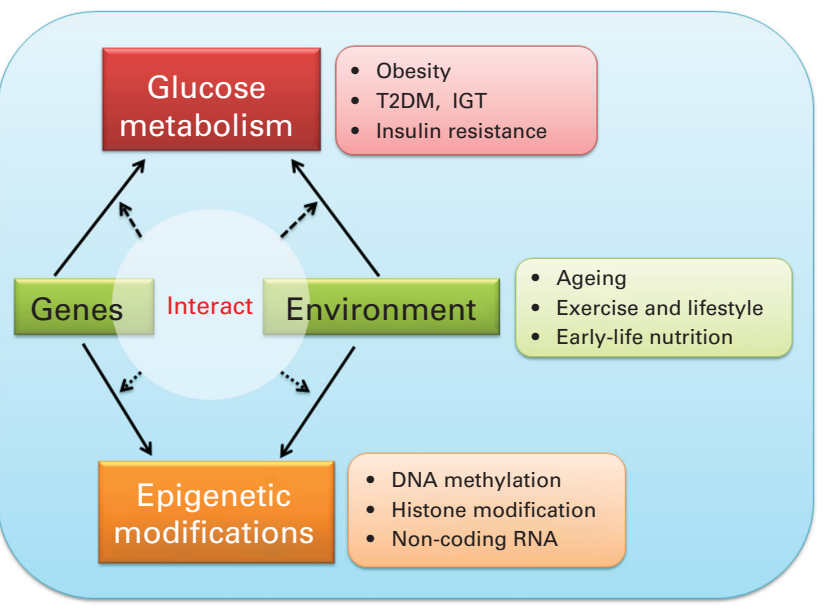

Fig. 1. Epigenetic modifications of early-life nutrition and glucose metabolism. Traditionally, genes together with some adult lifestyle factors determine the risk of developing some metabolic diseases such as obesity, type 2 diabetes mellitus (T2DM), impaired glucose tolerance (IGT) and insulin resistance in late life. However, there is now substantial evidence that prenatal and early-postnatal nutrition play a key role in determining susceptibility to these diseases. Epigenetics is likely to be the important molecular basis of malnutrition during early life and glucose metabolism disorders in later life. A colour version of this figure can be found online at http://www.journals.cambridge.org/bjn.

The term 'epigenetics' was first proposed by Waddington in 1942, and defined as a branch of biology that studied the causal interactions between genes and their products ${ }^{(4)}$. In 2006, the definition of epigenetics was updated and considered as a mechanism that could affect gene expression without altering the nucleotide sequence. Most importantly, it could be inherited between generations steadily by mitosis and meiosis through cell differentiation ${ }^{(5)}$. Epigenetic conditions can illustrate the reason why an organism produces many different cell types during its development, despite the fact that most of the cells in a multicellular organism share the same genetic information. Epigenetic modifications can regulate gene expression reversibly. The three major epigenetic processes are DNA methylation, histone modification and non-coding RNA. DNA methylation was the first recognised and the most well-characterised epigenetic modification in the $1970 \mathrm{~s}^{(6)}$. Histone modification can influence gene expression by altering chromatin structure ${ }^{(7)}$. Non-coding RNA, such as microRNA and long non-coding RNA, are also included in epigenetic modifications ${ }^{(8)}$. Epigenetic processes play a critical role in normal development and differentiation in mammals ${ }^{(9)}$. Epigenetics was defined by Skinner et al. ${ }^{(10)}$ in 2010 as 'molecular factors and processes around DNA that are mitotically stable and regulate genome activity independent of DNA sequence'. Although the definition of epigenetics has been updated constantly, the three core features are always reserved, namely (1) without any alterations in the DNA sequence, (2) heritability and (3) plasticity and reversibility. In the present review, we focus on DNA methylation.

\section{What is DNA methylation?}

DNA methylation is the first discovered and one important epigenetic modification. It is one of the best-studied epigenetic modifications in the context of altered environment. It is a biochemical process involving the covalent addition of a methyl group at the $5^{\prime}$ position of cytosine in DNA. This normally occurs on a cytosine followed by a guanine, known as a $\mathrm{CpG}$ dinucleotide (the $\mathrm{p}$ denotes the intervening phosphate group). It is catalysed by DNA methyltransferases, and $S$-adenosylmethionine is the methyl donor ${ }^{(11)}$. DNA methylation typically occurs in the context of $\mathrm{CPG}$ dinucleotides. $\mathrm{CPG}$ dinucleotides are not randomly distributed throughout the genome, but are often grouped in clusters at the $5^{\prime}$ regulatory regions of many genes, called $\mathrm{CpG}$ islands. Most $\mathrm{CpG}$ dinucleotides are methylated, but those located in $\mathrm{CpG}$ islands are usually unmodified ${ }^{(12)}$. However, hypermethylation of these CpG islands has a specific effect on reducing gene expression and repressing transcription, while their hypomethylation is related to transcriptional activation ${ }^{(13)}$ (Fig. 2). DNA methylation is a common modification in mammalian genomes. It constitutes a stable epigenetic symbol that can stably alter the expression of genes and transmit through DNA replication as cells divide and differentiate from embryonic stem cells into specific tissues $^{(11)}$. DNA methylation is essential for normal development and is associated with a number of key processes including genomic imprinting, X-chromosome inactivation ${ }^{(14)}$, suppression of repetitive elements and carcinogenesis ${ }^{(15)}$.

\section{Evidence of early-life nutrition and glucose metabolism from human cohorts}

\section{Fetal programming hypothesis}

In 1977 , the association between the quality of early-life environment and the future risk of disease in later life was first proposed by Forsdahl ${ }^{(16)}$, who discovered that infant mortality rate was positively associated with an increased risk of CVD in middle age. Subsequently, Barker et al. ${ }^{(17)}$ found an inverse relationship between birth weight and increased CVD mortality in 1989. The effects of early-life nutrition on the increased risk of metabolic diseases were most clearly shown in studies of the Dutch famine during the winter of 1944. These studies showed that individuals whose mothers were exposed to the famine in the late stage of gestation had lower birth weight and an increased risk of obesity, CVD, insulin resistance and hypertension in later life compared with unexposed individuals ${ }^{(18)}$. What is more, apart from nutrition deficiency in early life, overnutrition is also associated with a significantly increased susceptibility to

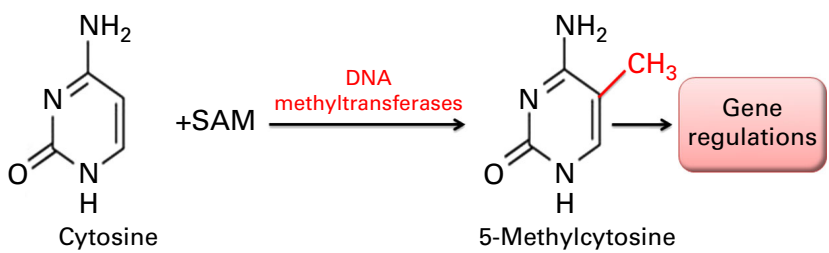

Fig. 2. Molecular mechanisms of DNA methylation. DNA methylation is a biochemical process involving the covalent addition of a methyl group at the $5^{\prime}$ position of cytosine in DNA. It is catalysed by DNA methyltransferases and $S$-adenosylmethionine (SAM) is the methyl donor. Hypermethylation or hypomethylation of some DNA can alter gene transcription. A colour version of this figure can be found online at http://www.journals.cambridge.org/bjn. 
metabolic diseases such as obesity, hypertension, atherosclerosis, insulin resistance and diabetes mellitus ${ }^{(19)}$. It can involve some organs related to glucose metabolism, including liver, pancreas, adipose tissue and skeletal muscle through different mechanisms (Fig. 3). Nutrition during early life has long-term phenotypic effects. This idea was first proposed by professor Barker and Hales in the 1990s, known as 'fetal programming hypothesis ${ }^{, 20)}$. After more than 20-year longterm research, it has now been accepted worldwide.

\section{Early-life nutrition and altered epigenetic modifications}

Epigenetic events are crucial for early development. However, it can be influenced by some environmental factors, potentially programming the genome for later adverse health outcomes. Gestation is the critical time window for maternal nutrition to affect the offspring. Early-life nutrition can induce persistent DNA methylation. More specifically, both undernutrition and overnutrition will bring about epigenetic modifications during early life, including embryonic development and the neonatal period. A number of clinical studies have shown that people born during the famine in The Netherlands still exhibited a lower methylation level of insulin-like growth factor 2 (IGF2) after 60 years, compared with those who were not exposed to prenatal famine ${ }^{(21)}$. It indicates that epigenetic modifications of some special genes affected by restricted periconceptional maternal nutrition can be persistent for decades. Another study has shown that children whose mothers took folic acid had $4.5 \%$ higher methylation in the IGF2 gene differentially methylated

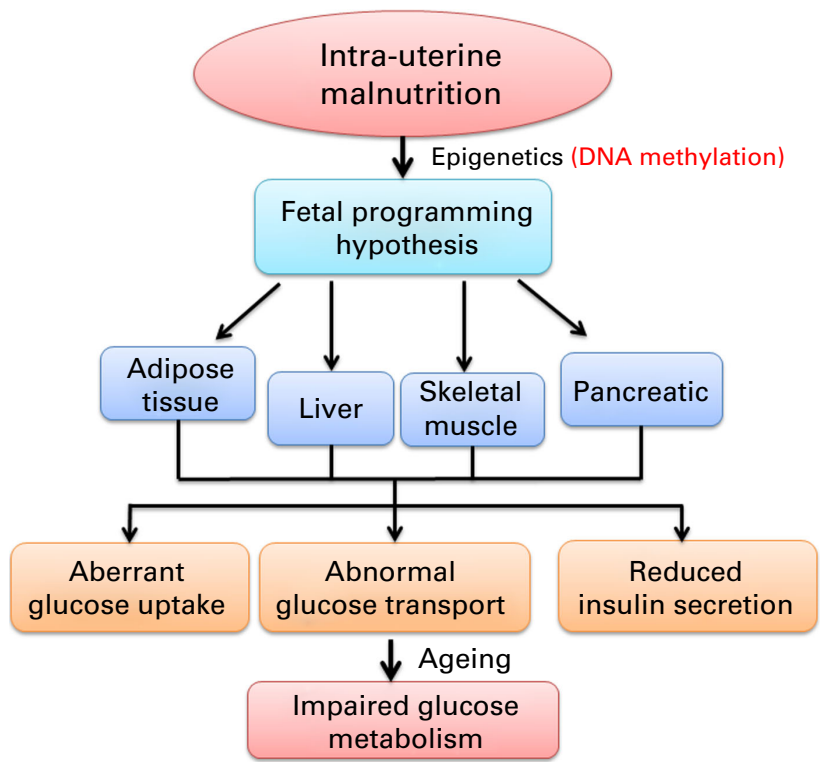

Fig. 3. Fetal programming hypothesis in relation to glucose metabolism. Nutrition during early life has long-term phenotypic effects along with ageing, such as impaired glucose metabolism. This idea was first proposed by professor Barker and Hales in the 1990s, known as 'fetal programming hypothesis'. It has been widely accepted that the essence of this hypothesis is epigenetic mechanism, such as DNA methylation. It can involve some organs related to glucose metabolism, including liver, pancreas, adipose tissue and skeletal muscle through different mechanisms. A colour version of this figure can be found online at http://www.journals.cambridge.org/bjn. region (DMR) than those whose mothers did not take folic acid. It also indicates an inverse independent association between IGF2 DMR and birth weight, which means that periconceptional folic acid use is associated with epigenetic changes in IGF2 in the child that may affect the intra-uterine programming of growth and development with consequences for health and disease throughout life ${ }^{(22)}$. A recent cohort study has demonstrated that retinoid $\mathrm{X}$ receptor- $\alpha$ (RXRA) methylation had an independent association with sex-adjusted childhood fat mass, which could explain more than $25 \%$ of the variance in childhood adiposity. Higher methylation of RXRA was associated with lower maternal carbohydrate intake in early pregnancy. These studies suggested that prenatal development is a substantial risk of metabolic diseases, such as diabetes, obesity and the metabolic syndrome ${ }^{(23)}$.

\section{Enlightenment of monozygotic twins}

Since epigenetic mechanisms are partly under genetic control $^{(24)}$, the role of epigenetic factors in disease aetiology could be confounded by genetic factors in unrelated individuals. Monozygotic (MZ) twins are well matched on their DNA sequence, thus providing an ideal model for epigenetic research. Type 1 diabetes mellitus shows an approximately 40\% concordant rate in $\mathrm{MZ}$ twins, suggesting the role of environmental factors and epigenetic modifications in the aetiology of the disease. Stefan et al. ${ }^{(25)}$ identified eighty-eight CpG sites displaying significant methylation changes in type 1 diabetes mellitusdiscordant $\mathrm{MZ}$ twin pairs. Using a well-matched MZ twin cohort, Zhao et al. ${ }^{(26)}$ demonstrated that global DNA methylation in peripheral blood leucocytes was significantly associated with insulin resistance. On average, a $10 \%$ increase in global DNA methylation was associated with a 4.55-unit increase in the homeostasis model assessment (HOMA) value. Some observational human and experimental animal studies have indicated that low birth weight was associated with an increased risk of metabolic diseases, such as obesity and T2DM. Although genetic factors might contribute to this association, studies of MZ twins showed that the twin who was lighter at birth had a more adverse metabolic profile in adulthood compared with its genetically identical co-twin, who was heavier at birth ${ }^{(27)}$. A population of twelve MZ twin pairs discordant for T2DM recognised a considerable non-genetic contribution to glucose metabolic disturbances in genetically identical individuals ${ }^{(28)}$. An exploratory approach showed that the site of umbilical cord insertion into the placenta in monochorionic twins has the strongest positive association with methylation in all IGF2/H19 $\mathrm{DMR}^{(29)}$, which was crucial for prenatal growth. In addition, the epigenetic state at this locus was environmentally labile. A study of 53- to 80-year-old MZ twin pairs discordant for T2DM found that increased variation in repetitive LINE1 DNA sequence methylation was associated with more phenotypic dissimilarity such as BMI and $2 \mathrm{~h}$ plasma glucose. T2DM-related genes, including PPARGC1 $\alpha$ encoding PPAR $\gamma$ co-activator $1 \alpha(P G C-1 \alpha)$ in muscle and hepatocyte nuclear factor- $4 \alpha(H N F 4 \alpha)$ in adipose tissue, have increased methylation in T2DM individuals ${ }^{(30)}$. A recent study on peri/postnatal epigenetic twins that contained a longitudinal cohort of 250 pairs of twins and their mothers 
confirmed that twins exhibit a wide range of epigenetic discordance at birth, and that within-pair birth-weight discordance correlated with epigenetic discordance in genes associated with glucose and lipid metabolism ${ }^{(31)}$. Therefore, all these lines of evidence support an epigenetic mechanism for the DOHaD and fetal programming hypothesis.

\section{Role of the placenta in developmental programming in the context of obesity and diabetes}

The placenta performs multiple functions required for bringing forth new life. It is not only a very important organ to provide nutrients for the developing fetus, it also exchanges a wide range of endocrine signals, cytokines and growth factors with the mother and the fetus, thereby regulating intra-uterine development. It is also well known that the placenta have a high and prolific expression of imprinted genes, and recent research have suggested that epigenetic modifications such as DNA methylation in imprinting and non-imprinting genes can have an important impact on the health of the offspring. Modified patterns of placental DNA methylation have been reported in human pregnancies. For example, it has been shown that the maternally imprinted mesoderm specific transcript (MEST) gene is hypomethylated in the placenta obtained from the newborns of mothers with gestational diabetes mellitus (GDM). In addition, decreased blood MEST methylation was also observed in adults with morbid obesity, suggesting that intra-uterine exposure to GDM has long-lasting effects on the epigenome of the offspring ${ }^{(32)}$. Another study showed that maternal glycaemia was associated with DNA methylation changes in leptin and adiponectin genes, suggesting that these epigenetic adaptations are probably functional and may have impacts on the long-term regulation of newborns' energy metabolism ${ }^{(33)}$.
Evidence of early-life nutrition and glucose metabolism from animal models

In addition to the evidence from studies of human cohorts, the association between dietary changes during specific windows of development and epigenomic modifications in later life has also been reported in several animal models. In the present review, we focus on the most widely studied challenges of nutrition, including maternal energy restriction (protein deficiency), maternal excessive energy (high fat feeding) and paternal malnutrition (protein deficiency and high-fat diet). We describe some well-known epigenetic changes that have been identified from animal models of obesity, insulin resistance and diabetes (see Table 1).

\section{Maternal energy restriction}

Protein restriction is frequently used as a model for maternal malnutrition. A low-protein (LP) diet is associated with impaired fetal growth, the development of obesity, insulin resistance and diabetes in the offspring ${ }^{(34)}$. Many epigenetic modifications have been reported in offspring exposed to a maternal LP diet. One of the first studies showing the association between nutritional imbalances during intra-uterine development and epigenetic modifications was that feeding a LP diet (9\% casein $v$. 18\% casein) to rats during pregnancy resulted in global DNA hypermethylation in the liver of the fetuses $^{(35)}$. Recent studies confirmed that maternal LP feeding during pregnancy might also result in locus-specific changes in DNA methylation. For example, feeding pregnant rats a protein-restricted diet induced hypomethylation of the glucocorticoid receptor $(G R)$ and $P P A R \alpha$ promoters in the liver of juvenile and adult offspring ${ }^{(36)}$. Also in Wistar rats, methylation of the PPAR $\alpha$ gene was $20.6 \%$ lower, with expression

Table 1. Summary of the relevant studies showing the effects of nutrients on DNA methylation in animal models

\begin{tabular}{|c|c|c|c|c|}
\hline Dietary conditions & Species & Regulated genes & Methylation & Reference \\
\hline Maternal LPD & Wistar rats & GR, PPAR $\alpha$ & Hypomethylation & Burdge et al..$^{(36)}$ \\
\hline Maternal LPD & Wistar rats & $H N F 4 \alpha$ & Hypermethylation & Sandovici et al. ${ }^{(38)}$ \\
\hline Maternal LPD & Balb/c mice & Leptin & Hypomethylation & Jousse et al. ${ }^{(39)}$ \\
\hline Maternal LPD & Meishan sows & Mitochondrial DNA & Hypomethylation & Vo et al. ${ }^{(40)}$ \\
\hline Maternal LPD & Piglet & HMGCR & Hypomethylation & Cong et al. ${ }^{(41)}$ \\
\hline Maternal energy restriction & Sheep & IGF2/H19 & Hypomethylation & Zhang et al. ${ }^{(42)}$ \\
\hline Maternal energy restriction & Sheep & $\mathrm{CpG}$ islands & Altered methylation & Sinclair et al. ${ }^{(43)}$ \\
\hline Maternal uterine artery ligation & SD rats & Gch1 & Hypermethylation & Thompson et al. ${ }^{(44)}$ \\
\hline Maternal HFD & C57BL/6J $\times$ DBA/2J hybrids & $\begin{array}{l}\text { MOR } \\
\text { Preproenkephalin }\end{array}$ & $\begin{array}{l}\text { Hypomethylation } \\
\text { Hypermethylation }\end{array}$ & Vucetic et al. ${ }^{(45)}$ \\
\hline Maternal HFD & C57BL/6J $\times$ DBA/2J hybrids & MOR & Hypermethylation & Vucetic et al. ${ }^{(46)}$ \\
\hline Maternal HFD & Mice & $\begin{array}{l}\text { Adiponectin } \\
\text { Leptin receptor }\end{array}$ & $\begin{array}{l}\text { Hypermethylation } \\
\text { Hypermethylation }\end{array}$ & Khalyfa et al. ${ }^{(47)}$ \\
\hline Maternal HFD & Mice & Leptin & Hypomethylation & Khalyfa et al. ${ }^{(47)}$ \\
\hline Maternal HFD & Mice & Global DNA & Altered methylation & Gallou-Kabani et al. ${ }^{(48)}$ \\
\hline Maternal HFD & Wistar rats & Placental DNA & Altered methylation & Kulkarni et al. ${ }^{(49)}$ \\
\hline Paternal HFD & SD rats & I/13ra2 promoter & Hypomethylation & $\mathrm{Ng}$ et al. ${ }^{(52)}$ \\
\hline Paternal LPD & C57BL/6J mice & $P P A R \alpha$ & Hypermethylation & Carone et al. ${ }^{(53)}$ \\
\hline Paternal LPD & Mice & Pik3ca & Hypermethylation & Wei et al. ${ }^{(54)}$ \\
\hline
\end{tabular}

$L P D$, low-protein diet; GR, glucocorticoid receptor; HNF4 $\alpha$, hepatocyte nuclear factor-4 $\alpha$; $H M G C R$, hepatic activation of 3-hydroxy-3-methylglutaryl-CoA reductase; IGF2/H19, insulin-like growth factor-2/H19; SD, Sprague-Dawley; Gch1, GTP cyclohydrolase 1; MOR, $\mu$-opioid receptor; HFD, high-fat diet; II13ra2, IL-13 receptor- $\alpha 2$; Pik3ca and Pik3r1, phosphatidylinositol 3-kinase subunits. 
being 10.5-fold higher, and that of the GR gene was $22 \cdot 8 \%$ lower, with expression being $200 \%$ higher in pups of dams that were fed a protein restriction diet throughout pregnancy ${ }^{(37)}$. The $H N F 4 \alpha$ gene has been implicated in the aetiology of T2DM. Sandovici et $a l .{ }^{(38)}$ observed that maternal LP diet during pregnancy and lactation could lead to progressive epigenetic silencing of the entire $H N F 4 \alpha$ locus in rat pancreatic islets, which weakened the promoter-enhancer interaction and resulted in a permanent reduction in $H N F 4 \alpha$ expression. Maternal protein undernutrition during pregnancy and lactation in Balb/c mice affected the balance between food intake and energy expenditure in adults. Moreover, this nutritional stress resulted in the removal of methyls at $\mathrm{CpG}$ sites located in the promoter of leptin, which was associated with DNA hypomethylation of the leptin promoter in adipose tissue $^{(39)}$. Interestingly, the DNA methylation of the LP diet is not restricted to rodents. Male offspring of maternal LP diet of Meishan sows during pregnancy and lactation demonstrated higher $G R$ binding to the mitochondrial DNA promoter, which was accompanied by lower cytosine methylation and hydroxymethylation on mitochondrial DNA promoter ${ }^{(40)}$. Similarly, piglets with maternal LP diet during pregnancy and lactation showed significantly lower body weight and liver weight at weaning. Hepatic activation of 3-hydroxy3-methylglutaryl-CoA reductase (HMGCR) gene transcription in LP piglets was associated with promoter hypomethylation ${ }^{(41)}$.

In addition to protein restriction, some other models of maternal energy restriction such as nutrient restriction can also affect the epigenome and the metabolism of the offspring. In one study, it has been shown that periconceptional undernutrition at least 5 months before conception to days 6 and 7 after conception was associated with a decrease in the adrenal mRNA expression of IGF2 and decreased methylation in the proximal CCCTC-binding factor (CTCF)-binding site in the DMR of the IGF2/H19 gene in the adrenal gland in offspring sheep, and it is associated with decreased expression of IGF2 in offspring along with an increase in the cortisol stress response in females ${ }^{(42)}$. In the fetal liver of sheep, maternal periconceptional restriction of vitamin $\mathrm{B}_{12}$, folate, and methionine altered DNA methylation of $4 \%$ of the $1400 \mathrm{CpG}$ islands examined, and more than half of these changes were specific to male offspring ${ }^{(43)}$. Bilateral uterine artery ligation of pregnant rats has been used to generate a model of fetal growth restriction. A genomewide survey of male Sprague-Dawley rat offspring showed altered DNA methylation of approximately 1400 loci that occurred predominantly in intergenic regions in pancreatic islets. Moreover, one gene in particular, GTP cyclohydrolase 1 (Gch1), showed almost a 3-fold reduction in mRNA expression, associated with hypermethylation at a conserved intergenic site $<45 \mathrm{~kb}$ upstream of the gene itself ${ }^{(44)}$. More importantly, these changes in DNA methylation may remain stable until adulthood, thus providing a molecular basis for DOHaD.

\section{Maternal energy excess}

Maternal energy restriction (LP diets) is not the only experimental model that suggests that maternal diet influences the epigenome of offspring during development. Nowadays, both the incidence of obesity during pregnancy and GDM are increasing along with improved living conditions. Therefore, scientists are increasingly concerned about the effect of overnutrition on human health. There is increasing evidence that energy excess in maternal diet is associated with an altered metabolic phenotype. Recently, studies have shown that maternal high-fat diet might modify DNA methylation and gene expression in the offspring. Maternal high-fat diet during gestation was associated with global and gene-specific promoter DNA hypomethylation, such as the dopamine reuptake transporter, the $\mu$-opioid receptor $(M O R)$ and preproenkephalin in the brain from the first-generation $\left(\mathrm{F}_{1}\right)$ offspring of $\mathrm{C} 57 \mathrm{BL} / 6 \mathrm{~J} \times \mathrm{DBA} / 2 \mathrm{~J}$ hybrids. These changes might affect feeding behaviour, which can increase obesity and obesity-associated risk for the metabolic syndrome ${ }^{(45)}$. During the postnatal period of the same model, the offspring of maternal high-fat diet showed increased binding of methyl CpG-binding protein $2(M e C P 2)$ to the $M O R$ promoter in reward-related regions of the brain, which can repress the transcription of the $M O R$ gene ${ }^{(46)}$. A recent study has shown that the offspring whose mothers fed on a high-fat diet during late gestation has increased weight accrual and food intake, exhibiting insulin resistance and hyperlipidaemia. Furthermore, the offspring mice emerged with increased methylation of adiponectin and leptin receptor, and decreased methylation of leptin genes ${ }^{(47)}$. Modified patterns of placental DNA methylation have also been reported in animal models exposed to an altered maternal environment. In one study, it has been shown that exposure to a high-fat diet during pregnancy triggered global and gene-specific DNA methylation in mouse placenta alterations in a manner dependent on the sex of the conceptus ${ }^{(48)}$. In Wistar rats, it has also been demonstrated that maternal intake of essential fatty acids during pregnancy affected the global levels of placental DNA methylation ${ }^{(49)}$.

Therefore, it is currently unknown whether these genes may contribute to the development of insulin resistance or diabetes in later life. It is certainly interesting to further explore the potential implication and epigenetic mechanisms of these genes on glucose metabolism.

\section{Paternal malnutrition}

Apart from the detrimental impacts of maternal malnutrition on abnormal glucose metabolism in offspring, recent studies have shown that paternal malnourished exposures could also affect the phenotype of the offspring. Paternal lifestyle and particular nutrition factors can affect spermatogenesis at the level of germ and sertoli cells ${ }^{(50)}$ and the composition of the seminal fluid ${ }^{(51)}$. Similarly, alterations in paternal diet are also associated with altered DNA methylation in the offspring. As shown by $\mathrm{Ng}$ et al. ${ }^{(52)}$, paternal exposure to a high-fat diet could induce intergenerational transmission of impaired glucose tolerance and insulin homeostasis in their female offspring. The IL-13 receptor- $\alpha 2$ (Il13ra2) promoter was hypomethylated in female offspring after high fat feeding of their fathers ${ }^{(52)}$. Offspring of fathers fed on a LP diet in C57BL/6J 
mice exhibited elevated hepatic expression of many genes involved in glucose metabolism and lipid biosynthesis. Epigenomic bisulphite sequence analysis on DNA from the liver of the offspring revealed numerous modest (about 20\%) changes in cytosine methylation depending on the paternal diet, including a substantial increase in methylation at an intergenic CpG island $50 \mathrm{~kb}$ upstream of the PPAR $\alpha$ gene $^{(53)}$. A recent study has shown that paternal prediabetes increased the susceptibility of the offspring to diabetes through gametic epigenetic alterations ${ }^{(54)}$. All these results indicate that paternal diet can also induce impaired glucose metabolism in offspring through epigenetic modification mechanisms, especially DNA methylation.

\section{Transgenerational epigenetic effects}

Maternal malnutrition during gestation and lactation not only have lifelong effects on $\mathrm{F}_{1}$ offspring, but can also impact the second-generation $\left(\mathrm{F}_{2}\right)$, despite the fact that there is no further dietary modification to $\mathrm{F}_{1}$. The first direct evidence of transgenerational epigenetic inheritance in mammals came from studies in mouse nucleocytoplasmic hybrids, showing that the cytoplasmic 'environment' of the early embryonic genome induces persistent and heritable changes in gene expression and $\mathrm{CpG}$ methylation ${ }^{(55)}$. Another study indicated that mice fed on a high-fat diet from adulthood developed T2DM themselves, which was reversible, but their $F_{1}$ and $F_{2}$ offspring developed T2DM irreversibly, without further high-fat diet feeding ${ }^{(56)}$. Similar results on glucose metabolism have been reported in another animal study on offspring of mothers with GDM. Impaired glucose tolerance was found in the $\mathrm{F}_{1}$-GDM mice, whose plasma glucose level was significantly increased, while the fasting insulin level of the $\mathrm{F}_{2}$-GDM was significantly increased. The expression of the imprinted genes IGF2 and H19 was down-regulated in pancreatic islets, caused by abnormal methylation status of the $\mathrm{DMR}^{(57)}$. Emerging evidence suggests that cross-generation transmission of adverse dietary effects during gestation/lactation is relevant to changes in epigenetics. Burdge et al. ${ }^{(58)}$ observed that DNA methylation of hepatic PPAR $\alpha$ in both $\mathrm{F}_{1}$ and $\mathrm{F}_{2}$ generations was reduced due to dietary protein restriction during gestation, despite that dietary protein restriction was only applied in one generation and no dietary modification was administered in their offspring. However, not all epigenetic changes are transmittable across generations. For example, diet-induced hypermethylation at agouti viable yellow is not inherited transgenerationally through the female ${ }^{(59)}$. A recent study uniquely revealed that prediabetes could be inherited transgenerationally through the mammalian germ line by DNA methylation ${ }^{(54)}$. In summary, most studies revealed that altered epigenetic modifications during early life could be transmitted to the next generation

In conclusion, studies of human cohorts and animal models demonstrated that developmental programming of adult diseases occurs at each nutrition spectrum during the early life, due to maternal energy deprivation, maternal nutritional excess and paternal malnutrition. Although the specific mechanisms leading to aberrant glucose metabolism in each situation are different, current evidence supports that epigenetic modifications, especially DNA methylation, may be an important molecular link between them, and this epigenome can be inherited from one generation to another.

\section{Developmental plasticity and epigenetic reversibility}

Generally speaking, plasticity is viewed as the ability that one genotype can produce different phenotypes in response to different environments. The time window of maximal plasticity appears to be during development, which is termed as 'Developmental plasticity'. It has evolved to provide the best chances of survival and reproductive success to the organism. Further studies illustrated that developmental plasticity could be regulated by epigenetic modifications. It was traditionally considered that epigenetic modifications were static in the control of gene expression. However, it is now being altered by the idea that these epigenomic marks are dynamically induced by some factors. Examples supporting the idea that intervention with drugs in early life can bring long-term benefits to health are presented as follows. Offspring born to dams with energy restriction during gestation and lactation are more likely to develop obesity and insulin resistance ${ }^{(60)}$. However, when leptin was given to neonatal mice born to dams that suffered from energy restriction, the offspring mice showed a phenotype close to healthy controls and did not develop obesity in later life even when fed with an energy-dense high-fat diet ${ }^{(61)}$. It suggests that developmental metabolic programming is potentially reversible by an intervention late in the phase of developmental plasticity. The long-lasting effects of early leptin intervention has been shown to be associated with a consistent up-regulation of some critical glucose metabolic genes such as the transcription factor $P P A R \alpha$, whereas a sustained up-regulation of $P P A R \alpha$ is regulated by reduced DNA methylation in the promoter region of $P P A R \alpha^{(62)}$. Stress responses in the adult rat are programmed early in life by maternal care, and are associated with altered transcription factor binding to the hippocampal $G R$ promoter ${ }^{(63)}$. Interestingly, central infusion of these adult offspring with L-methionine, a precursor to $S$-adenosylmethionine that serves as the donor of methyl groups for DNA methylation, with altered DNA methylation due to maternal behaviour programming during gestation can be reversed, together with altered nerve growth factor-inducible protein-A binding to the $G R$ promoter and hypothalamic-pituitaryadrenal and behavioural responses to stress ${ }^{(64,65)}$. These results demonstrate that despite the inherent stability of epigenomic marks established early in life through behavioural programming, they are potentially reversible in the adult brain. Therefore, in view of the reversibility of epigenetic mechanisms, intervention with drugs during early life may ameliorate glucose metabolism disorders in later life if the early disrupting environment has resulted in modified epigenetics, which can generate long-lasting effects.

\section{Conclusions}

In summary, the increased risk of aberrant glucose metabolism during later life is not only determined by the genotype, 
to some extent, it is also distinctly affected by early developmental nutrition. The fundamental mechanism is that early developmental nutrition can alter epigenetic modifications, especially DNA methylation, which, in turn, can alter the expression of some genes related to cell differentiation and glucose metabolism. Pregnancy and lactation are the pivotal periods of growth and development, which are very sensitive to the changes in developmental environments, such as nutrition. Therefore, it is very meaningful to study the epigenetics. It may be that the mechanisms account for the relationship between early-life nutrition and glucose metabolism in later life, which can help us explain the characteristics of the onset of some chronic diseases such as diabetes. More importantly, epigenetics may be utilised as a new theoretical foundation for the aetiology of diabetes mellitus, to explore a novel drug for the prevention and intervention of diabetes from the early stage of life in the future.

\section{Acknowledgements}

The present review was supported by the National Key Program of Clinical Science and the National Natural Science Foundation of China (NSFC) (project no. 81170736).

The authors' contributions are as follows: J. Z. and $X$. X. contributed to the idea, conception and design of the review; Q. Z. and M. Y. drafted and critically revised the manuscript for important intellectual content. All authors read and approved the final version.

The authors declare that there are no conflicts of interest.

\section{References}

1. International Diabetes Federation (2013) IDF Diabetes Atlas, 6th ed. http://www.idf.org/diabetesatlas

2. Slatkin M (2009) Epigenetic inheritance and the missing heritability problem. Genetics 182, 845-850.

3. Gluckman PD, Hanson MA, Buklijas T, et al. (2009) Epigenetic mechanisms that underpin metabolic and cardiovascular diseases. Nat Rev Endocrinol 5, 401-408.

4. Waddington $\mathrm{CH}$ (2012) The epigenotype. 1942. Int J Epidemiol 41, 10-13.

5. Holliday R (2006) Epigenetics: a historical overview. Epigenetics 1, 76-80.

6. Holliday R \& Pugh JE (1975) DNA modification mechanisms and gene activity during development. Science $\mathbf{1 8 7}$, 226-232.

7. Turner BM (1998) Histone acetylation as an epigenetic determinant of long-term transcriptional competence. Cell Mol Life Sci 54, 21-31.

8. Aguilera O, Fernandez AF, Munoz A, et al. (2010) Epigenetics and environment: a complex relationship. J Appl Physiol 109, 243-251.

9. Fraga MF (2009) Genetic and epigenetic regulation of aging. Curr Opin Immunol 21, 446-453.

10. Skinner MK, Manikkam M \& Guerrero-Bosagna C (2010) Epigenetic transgenerational actions of environmental factors in disease etiology. Trends Endocrinol Metab 21, 214-222.

11. Bird A (2002) DNA methylation patterns and epigenetic memory. Gene Dev 16, 6-21.

12. Gardinergarden M \& Frommer M (1987) CpG islands in vertebrate genomes. J Mol Biol 196, 261-282.
13. Reik W \& Dean W (2001) DNA methylation and mammalian epigenetics. Electrophoresis 22, 2838-2843.

14. Kacem S \& Feil R (2009) Chromatin mechanisms in genomic imprinting. Mamm Genome 20, 544-556.

15. Jaenisch R \& Bird A (2003) Epigenetic regulation of gene expression: how the genome integrates intrinsic and environmental signals. Nat Genet 33, 245-254.

16. Forsdahl A (1977) Are poor living conditions in childhood and adolescence an important risk factor for arteriosclerotic heart disease? Br J Prev Soc Med 31, 91-95.

17. Barker DJ, Winter PD, Osmond C, et al. (1989) Weight in infancy and death from ischaemic heart disease. Lancet 2 , 577-580.

18. Painter RC, Roseboom TJ \& Bleker OP (2005) Prenatal exposure to the Dutch famine and disease in later life: an overview. Reprod Toxicol 20, 345-352.

19. Curhan GC, Willett WC, Rimm EB, et al. (1996) Birth weight and adult hypertension, diabetes mellitus, and obesity in US men. Circulation 94, 3246-3250.

20. Hales CN, Barker DJP, Clark PMS, et al. (1991) Fetal and infant growth and impaired glucose-tolerance at age 64 . Brit Med J 303, 1019-1022.

21. Tobi EW, Lumey LH, Talens RP, et al. (2009) DNA methylation differences after exposure to prenatal famine are common and timing- and sex-specific. Hum Mol Genet 18, 4046-4053.

22. Steegers-Theunissen RP, Obermann-Borst SA, Kremer D, et al. (2009) Periconceptional maternal folic acid use of 400 microg per day is related to increased methylation of the IGF2 gene in the very young child. PLOS ONE 4, e7845.

23. Godfrey KM, Sheppard A, Gluckman PD, et al. (2011) Epigenetic gene promoter methylation at birth is associated with child's later adiposity. Diabetes 60, 1528-1534.

24. Chong S \& Whitelaw E (2004) Epigenetic germline inheritance. Curr Opin Genet Dev 14, 692-696.

25. Stefan M, Zhang W, Concepcion E, et al. (2013) DNA methylation profiles in type 1 diabetes twins point to strong epigenetic effects on etiology. J Autoimmun 50, 33-37.

26. Zhao J, Goldberg J, Bremner JD, et al. (2012) Global DNA methylation is associated with insulin resistance: a monozygotic twin study. Diabetes 61, 542-546.

27. Grunnet L, Vielwerth S, Vaag A, et al. (2007) Birth weight is nongenetically associated with glucose intolerance in elderly twins, independent of adult obesity. J Intern Med $\mathbf{2 6 2}$, 96-103.

28. Vaag A, Henriksen JE, Madsbad S, et al. (1995) Insulin secretion, insulin action, and hepatic glucose production in identical twins discordant for non-insulin-dependent diabetes mellitus. J Clin Invest 95, 690-698.

29. Loke YJ, Galati JC, Morley R, et al. (2013) Association of maternal and nutrient supply line factors with DNA methylation at the imprinted IGF2/H19 locus in multiple tissues of newborn twins. Epigenetics 8, 1069-1079.

30. Ribel-Madsen R, Fraga MF, Jacobsen S, et al. (2012) Genomewide analysis of DNA methylation differences in muscle and fat from monozygotic twins discordant for type 2 diabetes. PlOS ONE 7, e51302.

31. Loke YJ, Novakovic B, Ollikainen M, et al. (2013) The Peri/ postnatal Epigenetic Twins Study (PETS). Twin Res Hum Genet 16, 13-20.

32. El Hajj N, Pliushch G, Schneider E, et al. (2013) Metabolic programming of MEST DNA methylation by intrauterine exposure to gestational diabetes mellitus. Diabetes 62 , 1320-1328.

33. Houde AA, Hivert MF \& Bouchard L (2013) Fetal epigenetic programming of adipokines. Adipocyte 2, 41-46. 
34. Bocock PN \& Aagaard-Tillery KM (2009) Animal models of epigenetic inheritance. Sem Reprod Med 27, 369-379.

35. Rees WD, Hay SM, Brown DS, et al. (2000) Maternal protein deficiency causes hypermethylation of DNA in the livers of rat fetuses. J Nutr 130, 1821-1826.

36. Burdge GC, Slater-Jefferies J, Torrens C, et al. (2007) Dietary protein restriction of pregnant rats in the $\mathrm{F}_{0}$ generation induces altered methylation of hepatic gene promoters in the adult male offspring in the $\mathrm{F}_{1}$ and $\mathrm{F}_{2}$ generations. $\mathrm{BrJ}$ Nutr 97, 435-439

37. Lillycrop KA, Phillips ES, Jackson AA, et al. (2005) Dietary protein restriction of pregnant rats induces and folic acid supplementation prevents epigenetic modification of hepatic gene expression in the offspring. J Nutr 135, 1382-1386.

38. Sandovici I, Smith NH, Nitert MD, et al. (2011) Maternal diet and aging alter the epigenetic control of a promoterenhancer interaction at the $H n f 4 a$ gene in rat pancreatic islets. Proc Natl Acad Sci U S A 108, 5449-5454.

39. Jousse C, Parry L, Lambert-Langlais S, et al. (2011) Perinatal undernutrition affects the methylation and expression of the leptin gene in adults: implication for the understanding of metabolic syndrome. FASEB J 25, 3271-3278.

40. Vo TX, Revesz A, Sohi G, et al. (2013) Maternal protein restriction leads to enhanced hepatic gluconeogenic gene expression in adult male rat offspring due to impaired expression of the liver $X$ receptor. $J$ Endocrinol 218, 85-97.

41. Cong R, Jia Y, Li R, et al. (2012) Maternal low-protein diet causes epigenetic deregulation of $H M G C R$ and $C Y P 7 \alpha 1$ in the liver of weaning piglets. J Nutr Biochem 23, $1647-1654$

42. Zhang S, Rattanatray L, MacLaughlin SM, et al. (2010) Periconceptional undernutrition in normal and overweight ewes leads to increased adrenal growth and epigenetic changes in adrenal IGF2/H19 gene in offspring. FASEB $J$ 24, 2772-2782.

43. Sinclair KD, Allegrucci C, Singh R, et al. (2007) DNA methylation, insulin resistance, and blood pressure in offspring determined by maternal periconceptional B vitamin and methionine status. Proc Natl Acad Sci U S A 104, 19351-19356.

44. Thompson RF, Fazzari MJ, Niu H, et al. (2010) Experimental intrauterine growth restriction induces alterations in DNA methylation and gene expression in pancreatic islets of rats. J Biol Chem 285, 15111-15118.

45. Vucetic Z, Kimmel J, Totoki K, et al. (2010) Maternal high-fat diet alters methylation and gene expression of dopamine and opioid-related genes. Endocrinology 151, 4756-4764.

46. Vucetic Z, Kimmel J \& Reyes TM (2011) Chronic high-fat diet drives postnatal epigenetic regulation of $\mu$-opioid receptor in the brain. Neuropsychopharmacology 36, 1199-1206.

47. Khalyfa A, Carreras A, Hakim F, et al. (2013) Effects of late gestational high-fat diet on body weight, metabolic regulation and adipokine expression in offspring. Int $J$ Obes (Lond) 37, 1481-1489.

48. Gallou-Kabani C, Gabory A, Tost J, et al. (2010) Sex- and diet-specific changes of imprinted gene expression and DNA methylation in mouse placenta under a high-fat diet. PLOS ONE 5, e14398.
49. Kulkarni A, Dangat K, Kale A, et al. (2011) Effects of altered maternal folic acid, vitamin $\mathrm{B}_{12}$ and docosahexaenoic acid on placental global DNA methylation patterns in Wistar rats. PLOS ONE 6, e17706.

50. Sharpe RM (2010) Environmental/lifestyle effects on spermatogenesis. Philos Trans R Soc Lond B Biol Sci 365 $1697-1712$.

51. Robertson SA (2005) Seminal plasma and male factor signalling in the female reproductive tract. Cell Tissue Res $\mathbf{3 2 2}$ $43-52$.

52. Ng SF, Lin RC, Laybutt DR, et al. (2010) Chronic high-fat diet in fathers programs $\beta$-cell dysfunction in female rat offspring. Nature 467, 963-966.

53. Carone BR, Fauquier L, Habib N, et al. (2010) Paternally induced transgenerational environmental reprogramming of metabolic gene expression in mammals. Cell $\mathbf{1 4 3}$ 1084-1096.

54. Wei Y, Yang CR, Wei YP, et al. (2014) Paternally induced transgenerational inheritance of susceptibility to diabetes in mammals. Proc Natl Acad Sci U S A 111, 1873-1878.

55. Roemer I, Reik W, Dean W, et al. (1997) Epigenetic inheritance in the mouse. Curr Biol 7, 277-280.

56. Gniuli D, Calcagno A, Caristo ME, et al. (2008) Effects of high-fat diet exposure during fetal life on type 2 diabetes development in the progeny. J Lipid Res 49, 1936-1945.

57. Ding GL, Wang FF, Shu J, et al. (2012) Transgenerational glucose intolerance with $\mathrm{Ig} / 2 / \mathrm{H} 19$ epigenetic alterations in mouse islet induced by intrauterine hyperglycemia. Diabetes 61, 1133-1142.

58. Burdge GC, Hanson MA, Slater-Jefferies JL, et al. (2007) Epigenetic regulation of transcription: a mechanism for inducing variations in phenotype (fetal programming) by differences in nutrition during early life? Br J Nutr 97, 1036-1046.

59. Waterland RA, Travisano M \& Tahiliani KG (2007) Dietinduced hypermethylation at agouti viable yellow is not inherited transgenerationally through the female. FASEB J 21, 3380-3385.

60. Vickers $\mathrm{MH}$, Breier BH, Cutfield WS, et al. (2000) Fetal origins of hyperphagia, obesity, and hypertension and postnatal amplification by hypercaloric nutrition. Am J Physiol Endocrinol Metab 279, E83-E87.

61. Vickers MH, Gluckman PD, Coveny AH, et al. (2005) Neonatal leptin treatment reverses developmental programming. Endocrinology 146, 4211-4216.

62. Gluckman PD, Lillycrop KA, Vickers MH, et al. (2007) Metabolic plasticity during mammalian development is directionally dependent on early nutritional status. Proc Natl Acad Sci U S A 104, 12796-12800.

63. Weaver IC, Cervoni N, Champagne FA, et al. (2004) Epigenetic programming by maternal behavior. Nat Neurosci 7 , $847-854$

64. Weaver IC, Champagne FA, Brown SE, et al. (2005) Reversal of maternal programming of stress responses in adult offspring through methyl supplementation: altering epigenetic marking later in life. J Neurosci 25, 11045-11054.

65. Weaver IC (2007) Epigenetic programming by maternal behavior and pharmacological intervention. Nature versus nurture: let's call the whole thing off. Epigenetics 2, 22-28. 